\title{
Database of typical food portion sizes in Irish pre-school children aged 1-4 years
}

\author{
M. Giltinan, J. Lyons, J. Walton and A. Flynn \\ School of Food and Nutritional Sciences, University College Cork, Cork, Republic of Ireland
}

Typical food portion sizes have previously been reported for Irish children, adolescents and adults, ${ }^{(1)}$ but not for pre-school children. The aim of this study was to develop a database describing typical portion weights for a selection of commonly consumed foods for Irish pre-school children. Analysis was based on the National Pre-School Nutrition Survey (2010/2011) which was carried out to establish a database of habitual food and beverage consumption in a representative sample of Irish pre-school children $(n=500)$ aged $1-4$ years. A 4-d weighed food record was used to collect food intake data. Methods of quantifying food weights included: foods weighed by caregivers $(78 \%)$, food weights derived from manufacturers $(7 \%)$, use of age-appropriate photographic food atlases $(6 \%)^{(2)}$ and household measures $(6 \%)$.

Portion size was defined as the weight of food consumed per eating occasion i.e. weight served minus leftovers. The database describes the median, $25^{\text {th }}$ and $75^{\text {th }}$ percentile portion weights for approximately 100 food items. Typical (median) portion weights (g) and number of eating occasions $(n)$ are described below for a selection of commonly consumed foods for pre-school children, split by age (y).

\begin{tabular}{|c|c|c|c|c|c|c|c|c|}
\hline \multirow[b]{2}{*}{ Age } & \multicolumn{2}{|c|}{$1 \mathrm{y}(n=126)$} & \multicolumn{2}{|c|}{$2 \mathrm{y}(n-=124)$} & \multicolumn{2}{|c|}{$3 \mathrm{y}(n=126)$} & \multicolumn{2}{|c|}{$4 \mathrm{y}(n=124)$} \\
\hline & $n$ & median $(\mathrm{g})$ & $n$ & median $(\mathrm{g})$ & $n$ & median $(\mathrm{g})$ & $n$ & median $(\mathrm{g})$ \\
\hline Pasta, boiled & 103 & 51 & 95 & 68 & 79 & 71 & 115 & 81 \\
\hline White bread, sliced & 169 & 27 & 205 & 36 & 222 & 38 & 229 & 38 \\
\hline Brown bread, sliced & 101 & 27 & 175 & 34 & 141 & 36 & 126 & 38 \\
\hline RTEBC, dry & 351 & 18 & 379 & 20 & 418 & 24 & 425 & 25 \\
\hline Porridge (made up with milk/water) & 63 & 89 & 73 & 118 & 71 & 103 & 41 & 137 \\
\hline Milk, as a beverage & 817 & 189 & 616 & 160 & 436 & 138 & 404 & 140 \\
\hline Milk, on cereal & 301 & 88 & 330 & 96 & 347 & 100 & 372 & 100 \\
\hline Yoghurt, all types & 181 & 90 & 194 & 100 & 219 & 100 & 213 & 100 \\
\hline Fromage frais & 185 & 60 & 118 & 60 & 121 & 60 & 131 & 60 \\
\hline Cheese & 225 & 17 & 203 & 20 & 190 & 20 & 204 & 20 \\
\hline Potatoes (mashed/boiled) & 180 & 64 & 161 & 61 & 159 & 66 & 158 & 78 \\
\hline Carrots, boiled & 95 & 25 & 70 & 25 & 97 & 31 & 87 & 34 \\
\hline Peas, boiled & 54 & 12 & 30 & 15 & 32 & 21 & 27 & 22 \\
\hline Sausages & 62 & 40 & 77 & 40 & 69 & 45 & 73 & 41 \\
\hline Ham/luncheon meats & 64 & 18 & 105 & 20 & 152 & 20 & 170 & 20 \\
\hline Roast meat & 59 & 25 & 65 & 31 & 83 & 32 & 76 & 41 \\
\hline Fruit juice & 144 & 50 & 176 & 92 & 232 & 124 & 280 & 120 \\
\hline Bananas, flesh only & 227 & 59 & 186 & 83 & 191 & 81 & 167 & 90 \\
\hline Eating apples, edible part only & 103 & 40 & 154 & 65 & 150 & 70 & 172 & 77 \\
\hline Biscuits & 220 & 14 & 183 & 18 & 174 & 22 & 188 & 20 \\
\hline
\end{tabular}

RTEBC, ready-to-eat breakfast cereals.

Typical portion weights reported for 2-4 year olds were similar for most foods while those reported for 1 year olds were generally smaller. For foods which are generally packaged in unit amounts (e.g. yoghurt, fromage frais) or defined portions (e.g. sliced bread, sausages, sliced meats), typical portion weights were similar across age. This data may be useful for dietary assessment and dietary guidance for pre-school children.

The project was funded by the Irish Department of Agriculture, Food and the Marine under the Food for Health Research Initiative $2007-2012$.

1. Lyons J (2012) National Food Consumption Surveys of Children, Adolescents and Adults in Ireland: Food Portion sizes. PhD Thesis, University College Cork.

2. Foster E, Hawkins A \& Adamson A (2010) Young Person's Food Atlas: Pre-school. Food Standards Agency: London. 